\title{
FABRICATION OF MAGNETITE/PLA COMPOSITE NANOFIBER SHEET AND EVALUATION OF ITS MECHANICAL PROPERTIES
}

\author{
KAZUTO TANAKA, TAKUYA OKADA, YUTO MOTOHASHI \& TSUTAO KATAYAMA \\ Department of Biomedical Engineering, Doshisha University, Japan
}

\begin{abstract}
Due to special characteristics of nanofibers such as nanometer sized diameter, high surface area to volume ratio and higher alignment of molecular chains, nanofibers are expected to be widely used in many applications such as filter medias, drug delivery systems and scaffolds. Nanofibers containing magnetic nanoparticles have potential uses as functional nanofibers for many applications such as sensors and materials for drug delivery systems. As a material of nanofibers, due to their biocompatible and nontoxic characteristics, polylactic acid (PLA) and magnetite $\left(\mathrm{Fe}_{3} \mathrm{O}_{4}\right)$ can be used. In the fabrication process of nanofibers containing magnetic nanoparticles, controlling nanoparticle dispersion in nanofibers is one of the important issues to be overcome, and the effect of nanoparticle dispersion on the mechanical property of nanofibers has to be evaluated. In this study, three types of surface treatments, oleic acid treated, stearic acid treated and hot water treated are conducted to disperse magnetite in nanofibers. Random and aligned PLA nanofiber fabrics and magnetite/PLA composite nanofiber fabrics were fabricated by the electrospinning method, and their mechanical properties were revealed by tensile test. Oleic acid treatment was the most effective surface treatment. Highly aligned nanofibers can be fabricated by using a high rotation speed of drum collector. The tensile strength of PLA nanofibers and oleic acid treated magnetite/PLA composite nanofibers shows almost same values, although the other treatment has the lower tensile strength than PLA nanofibers. Aligned nanofibers tended to show high strengths.
\end{abstract}

Keywords: nanofiber, polylactic acid (PLA), magnetite, electrospinning, tensile test.

\section{INTRODUCTION}

Due to special characteristics of nanofibers such as nanometer sized diameter, high surface area to volume ratio and higher alignment of molecular chains [1], [2], nanofibers are expected to be widely used in many applications such as filter medias [3], drug delivery systems [4] and scaffolds [5]. Nanofibers containing magnetic nanoparticles have potential uses as functional nanofibers for many applications such as sensors and materials for drug delivery systems.

The electrospinning is a common method for the fabrication of nanofibers. In this process, when high-voltage is applied between polymer solution and the target, polymer solution was blown out from a needle hole. During the electrospinning process, the solvent evaporates and polymer nanofibers are fabricated on the target [1], [6].

As a material of nanofibers, due to their biocompatible [7] and nontoxic [8] characteristics, polylactic acid (PLA) and magnetite $\left(\mathrm{Fe}_{3} \mathrm{O}_{4}\right)$ can be used. In the fabrication process of nanofibers containing magnetic nanoparticles, controlling nanoparticle dispersion in the nanofibers is one of the important issues to be overcome. In recent research, the influence of surface treatment on nanoparticles on its dispersibility has been reported in films [9] and bulks [10] materials, but not in nanofibers. Suppression of agglomerate formations of magnetite is important when fabricating nanofibers containing magnetic nanoparticles. As, adding nanoparticles may affect its mechanical properties [11], it is necessary to evaluate the mechanical properties of nanofibers and the effect of nanoparticle dispersion on the mechanical property of nanofibers has to be evaluated. 
In this study, three types of surface treatments, oleic acid treated, stearic acid treated and hot water treated, are conducted to disperse magnetite in nanofibers. Random and aligned PLA nanofiber fabrics and magnetite/PLA composite nanofiber fabrics were fabricated by the electrospinning method, and their mechanical properties were revealed by tensile test.

\section{MECHANICAL PROPERTIES OF NANOFIBERS}

\subsection{Materials and the preparation process of solutions}

PLA (polylactic acid, Mw: 23000) was used for the materials of nanofibers. Magnetite $\left(\mathrm{Fe}_{3} \mathrm{O}_{4}\right.$, Toda Kogyo Corp.) with $170 \mathrm{~nm}$ in average particle diameters was used as superparamagnetic nanoparticles. Dichloromethane (DCM) and dimethyl-formamide (DMF) were used as the dissolution solvent (DCM: DMF, 8:2).

The PLA solution was prepared by dissolving of $10 \mathrm{wt} \%$ PLA in a solvent of DCM under stirring at $30^{\circ} \mathrm{C}$ for 60 minutes. Then, DMF was added to the solution and it was stirred at $30{ }^{\circ} \mathrm{C}$ for 5 minutes. In the preparation of magnetite/PLA solutions process, the magnetite solution was prepared by adding magnetite ( $20 \mathrm{wt} \%$, mass of magnetite to PLA) to DCM, and it was ultra-sonicated. Then, PLA solution and magnetite solution were mixed, and it was ultra-sonicated. Finally, adding DMF into the solution and ultra-sonicated it.

In this study, for dispersion of magnetite, the hot water treatment and surface treatments by the oleic acid treatment and the stearic acid treatment were used $\left(10 \mathrm{~mol} / \mathrm{m}^{3}\right.$ for solution). Magnetite nanoparticles adsorbed hydroxyls in hot water treatment in which magnetite is kept in hot water $\left(100^{\circ} \mathrm{C}\right)$ for 48 hours. Magnetite nanoparticles adsorbed the oleic acid and the stearic acid in the oleic acid treatment and the stearic acid treatment, respectively.

\subsection{Fabrication method of random and aligned nanofibers}

PLA and magnetite/PLA solutions were loaded into a plastic syringe equipped with a syringe needle. A high voltage was applied between the needle and the collector to fabricate PLA and magnetite/PLA composite nanofibers. A voltage of $10 \mathrm{kV}$, with a tip-collector distance of $150 \mathrm{~mm}$, at a feeding rate of $0.83 \times 10^{-5} \mathrm{~mm} / \mathrm{sec}(0.05 \mathrm{~mm} / \mathrm{min})$ was applied to fabricate nanofibers.

Fig. 1 shows the schematic drawing of the electrospinning process with the plate collector and the drum collector. Aligned PLA nanofibers and oleic acid treated magnetite/PLA composite nanofibers were fabricated by various rotation speed of the drum collector (500 rpm, $1000 \mathrm{rpm}, 1500 \mathrm{rpm}$, and $2000 \mathrm{rpm})$. For morphology observation by scanning electron microscope (SEM JSM-6390LT, JOEL Ltd., Japan), nanofibers were Pt-coated using sputter coating by Auto fine coater (LFL-1600, JOEL Ltd., Japan).

\subsection{Tensile test methods}

The following four types of random nanofibers were used for tensile test: PLA nanofibers, untreated magnetite/PLA composite nanofibers, oleic acid treated magnetite/PLA composite nanofibers and stearic acid treated magnetite/PLA composite nanofibers. The following two types of aligned nanofibers were used for tensile test: PLA nanofibers and oleic acid treated magnetite/PLA composite nanofibers.

Figs 2 and 3 show the schematic drawing of the tensile test specimen and the procedure of preparation of tensile test specimen. Tensile test specimens were prepared by punching tool, nanofibers with a sealing frame of polyester film and it was glued to another frame. The cross section of nanofibers which was used for calculation of stress was calculated by 
the thickness (measured with micro meter) and the width (measured with Vernier caliper) of the tensile test specimen. Tensile tests were conducted at a displacement rate of 0.017 $\mathrm{mm} / \mathrm{sec}(1 \mathrm{~mm} / \mathrm{min})$ by using the universal testing machine (EZ-graph, Shimadzu Co., Japan) equipped with $5 \mathrm{~N}$ load cells.

\subsection{Result and discussion}

\subsubsection{Observation of nanofibers}

Fig. 4 shows the SEM image of the magnetite/PLA composite nanofiber. Magnetite was aggregating in untreated magnetite/PLA composite nanofibers. Magnetite were dispersed more in stearic acid treated and hot water treated magnetite/PLA composite nanofibers compared to untreated one, and the oleic acid treated one showed the best dispersed condition. Fig. 5 shows the histogram of cluster size of magnetite. The frequency of big cluster size was high in untreated magnetite/PLA composite nanofibers. The frequency of small size was higher in oleic acid treated magnetite/PLA composite nanofibers. These results indicated that the oleic acid treatment is good for dispersing magnetite in nanofibers.

Figs 6 and 7 show the SEM image of aligned magnetite/PLA composite nanofibers and the histogram of fiber orientation angle of nanofibers. Nanofibers were oriented to the degree direction as the rotation speed of the drum collector was raised. More than 70 percent of nanofibers were oriented from 80 to 90 degrees at rotation speed of $2000 \mathrm{rpm}$.

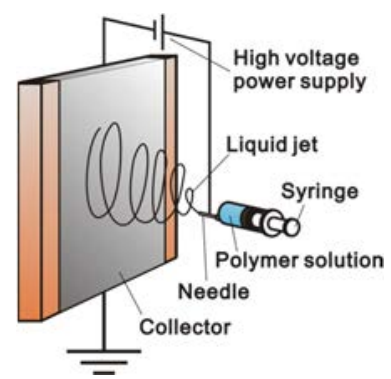

(a) The plate collector

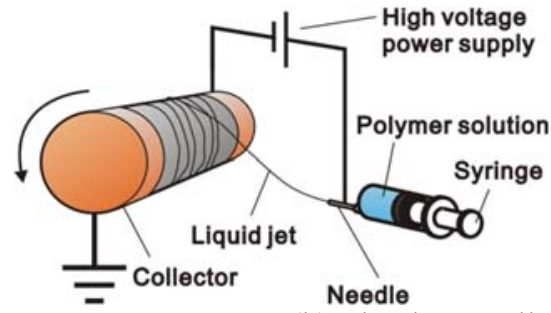

(b) The drum collector

Figure 1: Schematic drawing of electrospinning process.

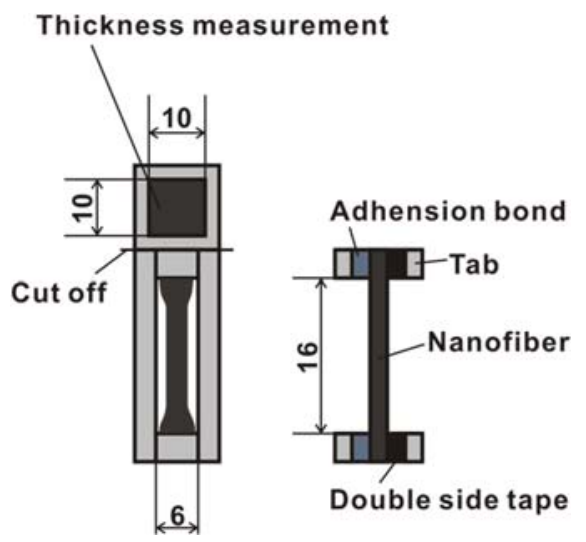

Figure 2: Tensile test specimen. 


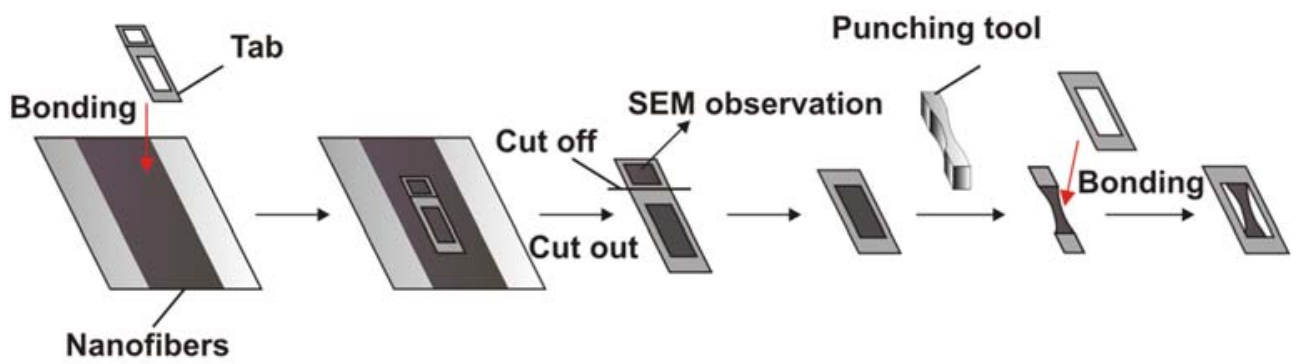

Figure 3: Procedure of preparation of tensile test specimen.

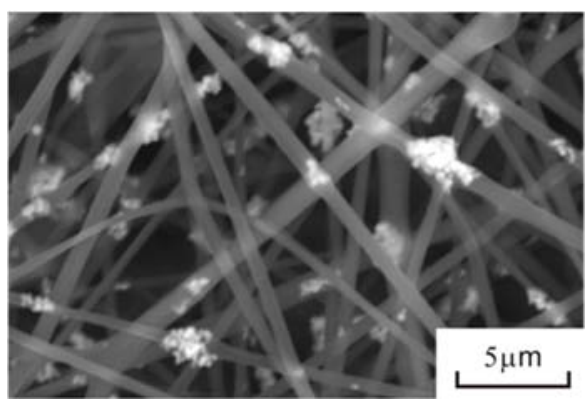

(a) Untreated

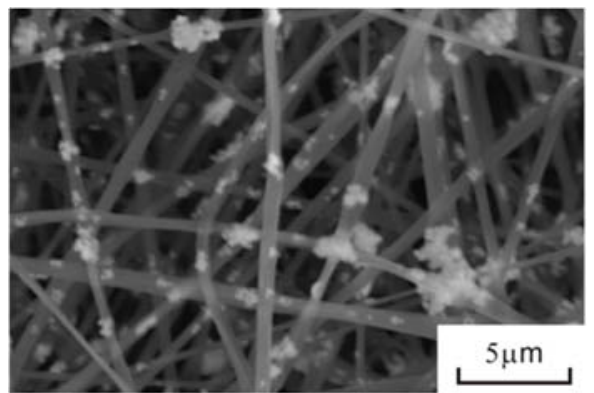

(c) Stearic acid treated

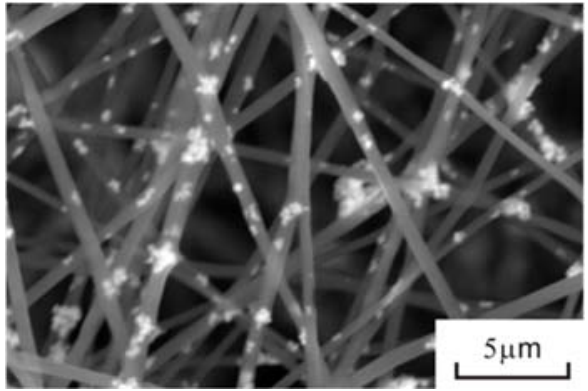

(b) Oleic acid treated

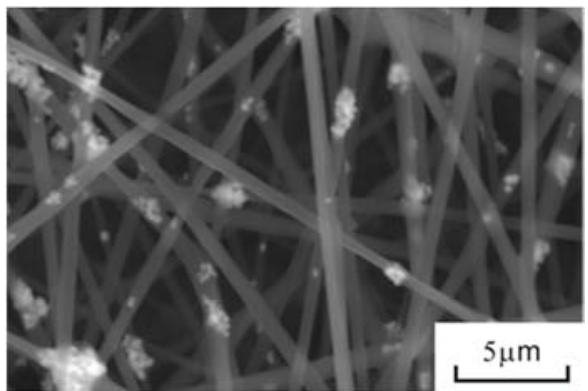

(d) Hot Water treated

Figure 4: SEM images of magnetite /PLA composite nanofibers.

\subsubsection{Tensile test of nanofibers}

Fig. 8 shows the stress-strain curves and tensile strength of four types of random nanofibers. The result shows that the tensile strength of PLA nanofibers and oleic acid treated magnetite/PLA composite nanofibers shows almost same value, although the other treated magnetite/PLA composite nanofibers has lower tensile strength than PLA nanofibers.

Fig. 9 shows the stress-strain curves and tensile strength of random and aligned nanofibers of PLA nanofibers and oleic acid treated magnetite/PLA composite nanofibers. Aligned nanofibers tended to show higher strengths than random nanofibers. 


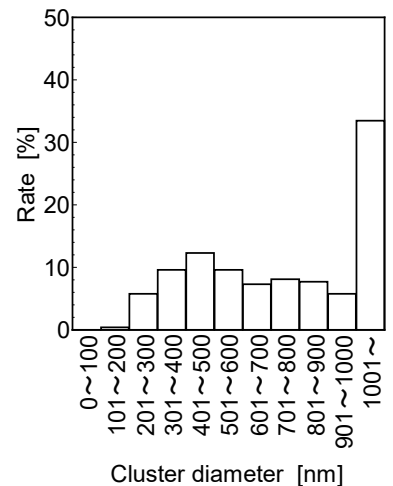

(a) Untreated

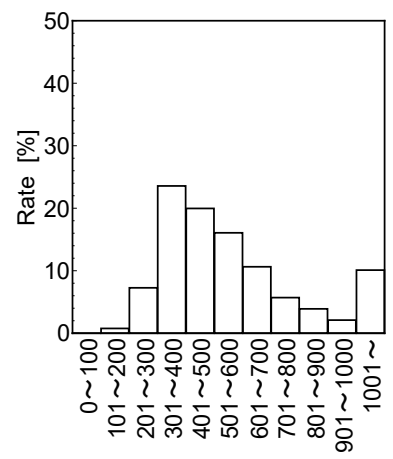

Cluster diameter [nm]

(c) Stearic acid treated

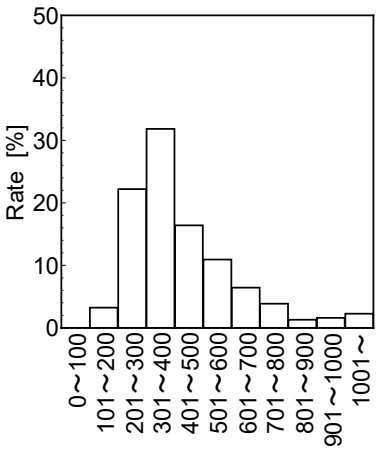

Cluster diameter [nm]

(b) Oleic acid treated

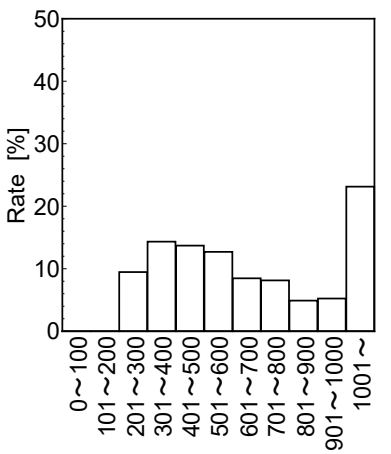

Cluster diameter $[\mathrm{nm}]$

(d) Hot Water treated

Figure 5: Cluster size distribution of magnetite.

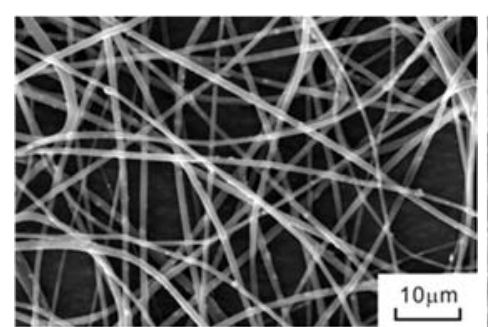

(a) $500 \mathrm{rpm}$

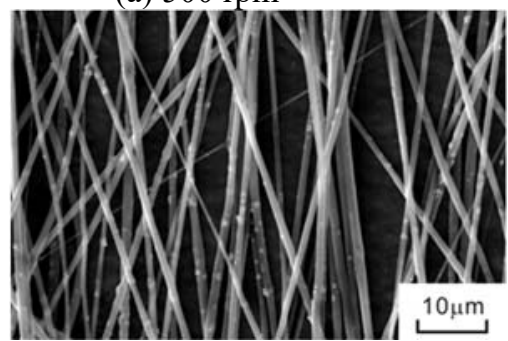

(c) $1500 \mathrm{rpm}$

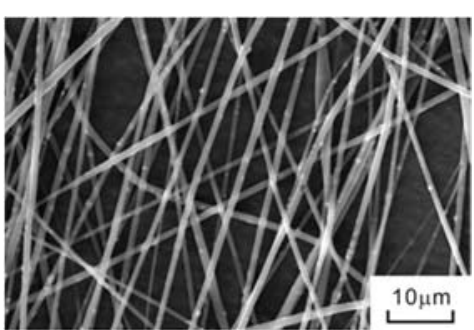

(b) $1000 \mathrm{rpm}$

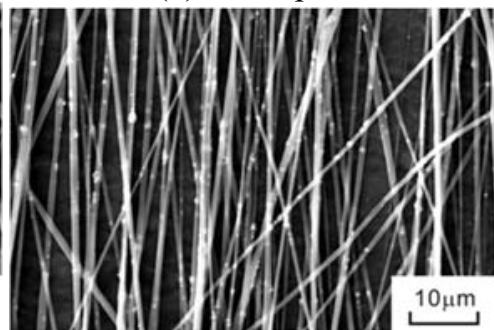

(d) $2000 \mathrm{rpm}$

Figure 6: SEM images of magnetite/PLA composite nanofibers. 


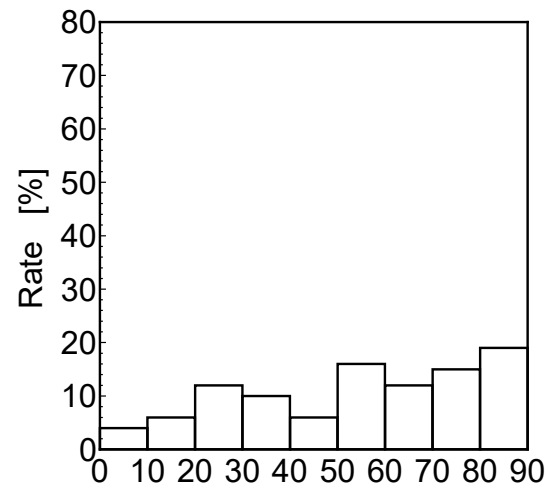

Fiber orientation angle [deg]

(a) $500 \mathrm{rpm}$

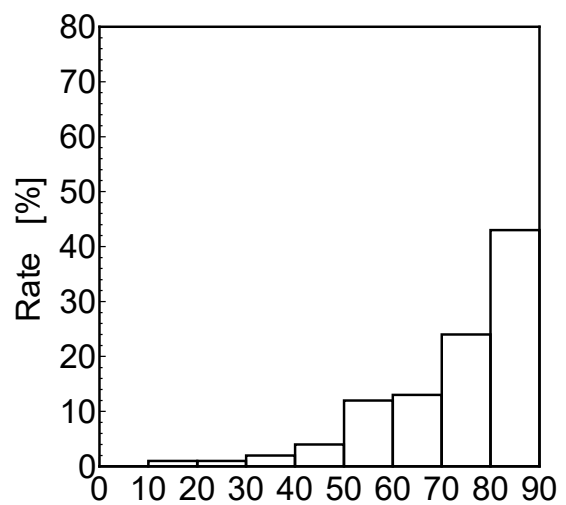

Fiber orientation angle [deg]

(c) $1500 \mathrm{rpm}$

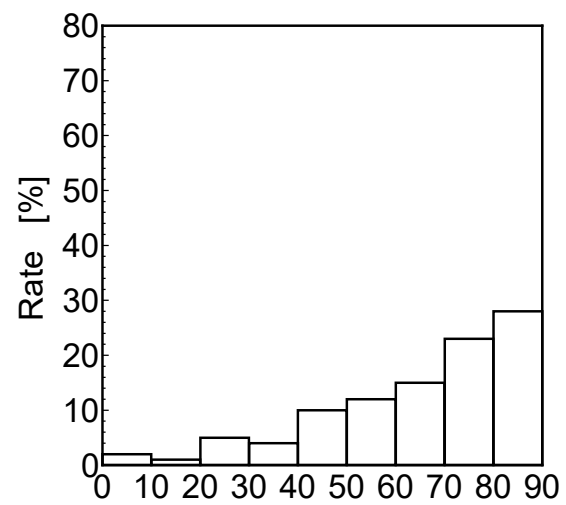

Fiber orientation angle [deg]

(b) $1000 \mathrm{rpm}$

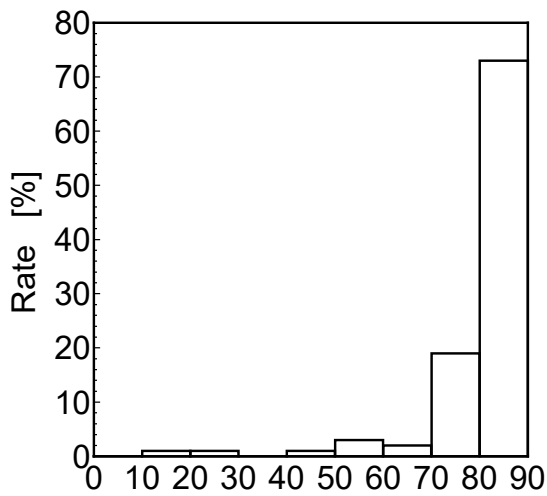

Fiber orientation angle [deg]

(d) $2000 \mathrm{rpm}$

Figure 7: Orientation angle of aligned magnetite/PLA composite nanofibers.

\section{CONCLUSION}

In this study, three types of surface treatments, oleic acid treated, stearic acid treated and hot water treated, are conducted to disperse magnetite in nanofibers. Random and aligned PLA nanofibers and magnetite/PLA composite nanofibers were fabricated by the electrospinning method, and their mechanical properties were revealed by tensile test. The investigation yielded the following conclusions.

1. Oleic acid treatment was the most effective surface treatment to disperse magnetite in PLA nanofibers.

2. Highly aligned nanofibers can be fabricated by using a high rotation speed of drum collector.

3. The tensile strength of PLA nanofibers and oleic acid treated magnetite/PLA composite nanofibers shows almost same value, although the other treated magnetite/PLA composite nanofibers has lower tensile strength than PLA nanofibers.

4. Aligned nanofibers tended to show higher strengths than random nanofibers. 


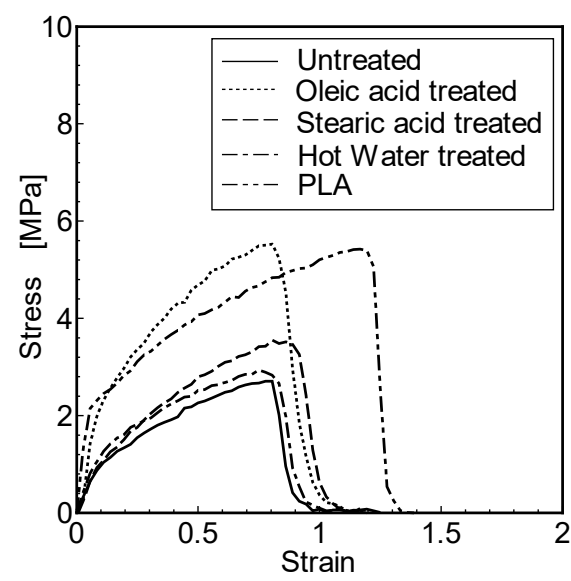

(a) Stress-strain curves

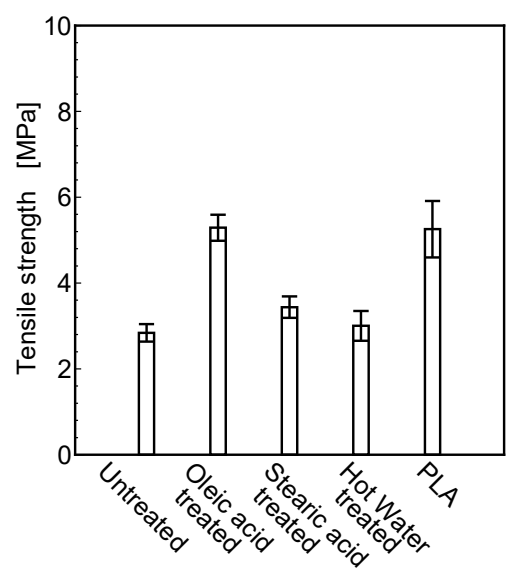

(b) Tensile strengths

Figure 8: Result of tensile test of random nanofibers.

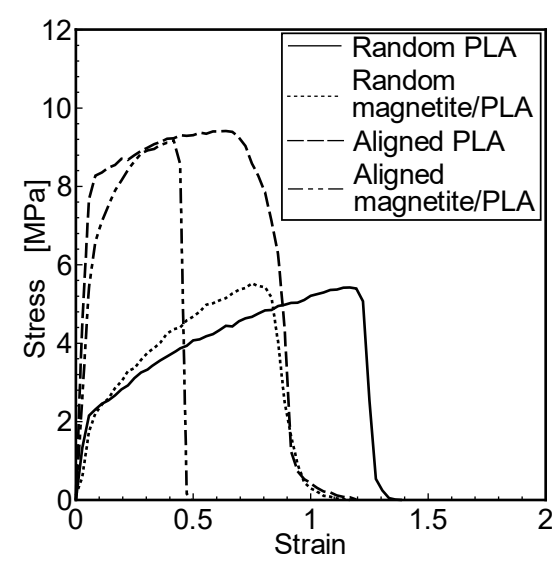

(a) Stress-strain curves

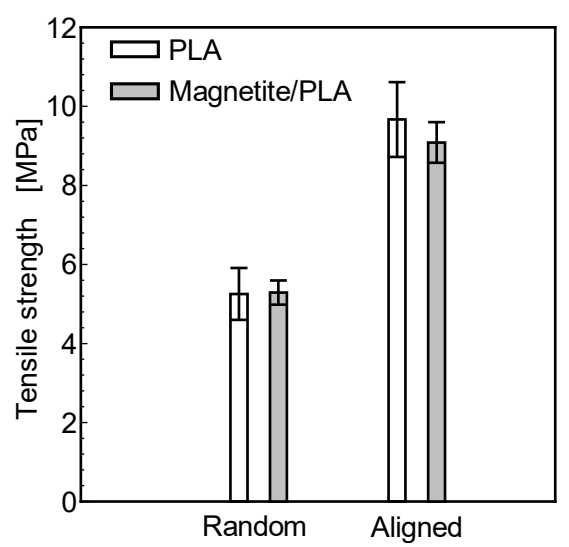

(b) Tensile strengths

Figure 9: Result of tensile test of random and aligned nanofibers of PLA nanofibers and oleic acid treated magnetite/PLA composite nanofibers.

\section{REFERENCES}

[1] Huang, M.Z., Zhang, Z.Y., Kotaki, M. \& Ramakrishna, S., A review on polymer nanofibers by electrospinning and their applications in nanocomposites. Composite science and technology, 63, pp. 2223-2253, 2003.

[2] Motomiya, M., Nanofiber to understand well, Nikkan Kogyo Shimbun, LTD, pp. 1248, 2006.

[3] Ramakrishna, S., Fujihara, K., Teo, W.E., Yong, T., Ma, Z. \& Ramaseshan, R., Electrospun nanofibers: solving global issues. Materials today, 9(3), pp. 40-50, 2006. 
[4] Yu, H., Yang, P., Zhang, Y., Ye, Q. \& Zeng, S., Regulation of biphasic drug release behavior by graphene oxide in polyvinyl pyrrolidone/poly ( $\varepsilon$-caprolactone) core/sheath nanofiber mats. Colloids and surfaces B: biointerfaces, 146, pp. 63-69, 2016.

[5] Ramalingam, M. et al., Nanofiber scaffold gradients for interfacial tissue engineering. Journal of biomaterials applications, 27(6), pp. 695-705, 2012.

[6] Ishii, Y., Sakai, H. \& Murata, H., A new electrospinning method to control the number and a diameter of uniaxially aligned polymer fibers. Materials letter, 62, pp. 33703372, 2008.

[7] Kawashima, N., A development of polylactic acid as bio-based polymers. Journal of synthetic organic chemistry, Japan, 61(5), pp. 496-505, 2003.

[8] Conn, E.R. et al., Safety assessment of polylactide (PLA) for use as a food-contact polymer. Food and chemical toxicology, 33(4), pp. 273-283, 1995.

[9] Kim, S., Hyun, K., Struth, B., Ahn, H.K. \& Clasen, C., Structural development of nanoparticle dispersion during drying in polymer nanocomposite films. Macromolecules, 49(23), pp. 90689079, 2016.

[10] Yang, Y., Lan, J. \& Li, X., Study on bulk aluminium matrix nanocomposite fabricated by ultrasonic dispersion of nanosized $\mathrm{SiC}$ particles in molten aluminium alloy. Materials science and engineering: $A, \mathbf{3 8 0}(1-2)$, pp. 378-383, 2004.

[11] Kim, W.H, Song, H.J. \& Kim, E.H., Nanofiber generation of gelatin-hydroxyapatite biomimetics for guided tissue regeneration. Advanced functional materials, 15, pp. 1988-1994, 2005. 